ISSN 0103-5150

Fisioter. Mov., Curitiba, v. 23, n. 2, p. 289-299, abr./jun. 2010

Licenciado sob uma Licença Creative Commons

\title{
Exercício físico no tratamento e prevenção de idosos com osteoporose: uma revisão sistemática
}

\author{
Physical exercise in the treatment and prevention of elderly with osteoporosis: \\ a systematic review
}

\author{
Marcelo Lasmar dos Santos ${ }^{[a]}$, Grasiely Faccin Borges ${ }^{[\mathrm{b}]}$ \\ [a] Acadêmico de Fisioterapia, bolsista de Iniciação Científica (FAPEAM), Universidade Federal do Amazonas (UFAM), Coari, AM - \\ Brasil, e-mail: marcelo_lasmar@hotmail.com \\ [b] Doutoranda em Ciências do Desporto pela Universidade de Coimbra, docente do Instituto de Saúde e Biotecnologia, Universidade \\ Federal do Amazonas (UFAM), Coari, AM - Brasil, e-mail: gfborges@ufam.edu.br
}

\section{Resumo}

Introdução: A atividade física influencia a manutenção das atividades ósseas normais, e por este motivo vem sendo indicada no tratamento da osteoporose. Objetivo: Verificar na literatura científica a influência do exercício físico na prevenção e no tratamento da osteoporose em idosos. Metodologia: A presente pesquisa foi sustentada através de revisão sistemática de literatura, com a utilização das bases de dado eletrônicas: LILACS, SciELO e PubMed. Foram utilizados os seguintes descritores na língua portuguesa: osteoporose, exercício físico, idosos, tratamento e prevenção, sendo estes utilizados para pesquisa nas bases LILACS e SciELO; e na língua inglesa, foram utilizados os seguintes: osteoporosis, physical exercise, elderly, prevention e treatment, sendo esta busca realizada na base de dados PubMed. Foram selecionados os resumos que apresentaram critérios iniciais de seleção. Resultados: De um total de 203 artigos encontrados, 27 preencheram os critérios de inclusão e exclusão, sendo que 9 artigos se concentraram no tratamento, 16 na prevenção e 2 no tratamento e na prevenção. Os exercícios que mais se destacaram no tratamento da osteoporose foram os seguintes: exercícios de extensão isométrica de tronco, exercícios em cadeia cinética aberta, coordenação e equilíbrio. Já na prevenção, os mais evidenciados foram: exercícios físicos de alta intensidade, exercícios de alto impacto e exercícios aeróbios. Conclusão: Pode-se concluir que o exercício físico é dado como fator importante, tanto na prevenção quanto no tratamento do idoso acometido pela osteoporose, e que ele possui especificidades de acordo com o objetivo a ser alcançado.

Palavras-chave: Exercício físico. Osteoporose. Idoso. 


\begin{abstract}
Introducion: Physical activity influences the maintenance of osseous normal activities, and for this reason has been indicated for the treatment of osteoporosis. Objective: To verify in the scientific literature the influence of physical exercise in prevention and treatment of osteoporosis in the elderly. Methodology: This research was supported though systematic review of literature with extensive use of the electronic database LILACS, SciELO and PubMed, with the descriptors osteoporosis, physical exercise, elderly, prevention and treatment. Were selected the abstracts that presented the initial criteria of selection. Results: From a total of 209 studies found, 27 met the criteria of inclusion and exclusion, where 9 studies focused on treatment, 16 on the prevention and 2 on the both prevention and treatment. The exercises that are most prominent in the treatment of osteoporosis were: isometric extension of the trunk, open kinetic-chain exercise, coordination and balance exercises. To the prevention, the exercises highlighted were: high-intensity exercises, high-impact exercises and aerobic exercises. Conclusion: Physical exercise is an important factor in both prevention and treatment of the elderly affected by osteoporosis disease, and the specificities of the exercise must be according to the objectives.
\end{abstract}

Keywords: Physical exercise. Osteoporosis. Elderly.

\title{
Introdução
}

As transformações ocorridas no século XX têm produzido impacto na estrutura etária da população e na distribuição quanto à morbimortalidade, o que exige mudanças nas respostas de cada sociedade em relação aos problemas de saúde. A redução da fecundidade e o aumento da expectativa de vida resultam no envelhecimento da população e aumento das taxas de doenças crônico-degenerativas, entre as quais a osteoporose $(1,2)$. Segundo o Instituto Brasileiro de Geografia e Estatística (IBGE), há uma estimativa de crescimento no número de idosos de cerca de 25 milhões de pessoas em 2020, dos quais aproximadamente 15 milhões do sexo feminino (3).

A osteoporose é uma doença metabólica do tecido ósseo, caracterizada por perda gradual da massa óssea, que enfraquece os ossos por deterioração da microarquitetura tecidual óssea, tornando-os mais frágeis e suscetíveis a fraturas (4). A perda da independência funcional, decorrente da incapacidade de deambular, é a principal consequência da fratura de quadril, seja por limitação funcional ou por medo de quedas. A inatividade física leva à piora da osteoporose e aumenta ainda mais os riscos de quedas e novas fraturas (5).

Segundo a Organização Mundial de Saúde (OMS), 1/3 das mulheres brancas acima de 65 anos são portadoras da osteoporose (6). Apesar de ser uma doença predominante em mulheres, ela também atinge os homens, estimando-se que cerca de $1 / 5$ dos homens brancos acima de 60 anos têm 25\% de chance de adquirir uma fratura osteoporótica.

A atividade física ou a prática regular de exercícios físicos influenciam a manutenção das atividades normais ósseas, e por este motivo a atividade física vem sendo indicada no tratamento da osteoporose (7). Entretanto, a relação entre atividade física, exercício físico e osteoporose tem levado pesquisadores a abordar várias discussões sobre este assunto, buscando melhor conhecimento sobre fatores como a intensidade, frequência e duração dos exercícios utilizados como método de prevenção e tratamento da patologia (8).

Pretendeu-se, através desta investigação, verificar na literatura científica a influência do exercício físico na prevenção e no tratamento da osteoporose em idosos, bem como apresentar os tipos de exercícios, volume e intensidade, a fim de oferecer subsídios para possíveis alternativas terapêuticas, com o intuito de contribuir para o desenvolvimento de técnicas na área de geriatria e gerontologia. 


\section{Metodologia}

A presente investigação foi sustentada através de uma revisão sistemática de literatura, com a utilização das bases de dados eletrônicos National Library of Medicine (MEDLINE/PubMed), Literatura LatinoAmericana e do Caribe em Ciência da Saúde (LILACS) e Scientific Electronic Library Online (SciELO).

A seleção dos artigos científicos estabeleceu os seguintes critérios: apresentar como objeto de estudo idosos acometidos por osteoporose, conter informações sobre a prática de atividade física e/ou exercício físico, e terem sido publicados a partir do ano 2000,

Os procedimentos foram organizados na seguinte sequência: na primeira etapa, realizou-se um levantamento de artigos encontrados com os descritores propostos; na segunda, ocorreu uma leitura e seleção criteriosa dos artigos para a formação de um banco de dados sistematizado. Os dados de todos os artigos incluídos foram coletados e armazenados em novo banco de dados, além das características sócio-demográficas da população estudada: país, ano, tipo de estudo, formas de coletas de dados, autores, entre outras variáveis que se mostraram interessantes para a investigação. Os artigos foram agrupados por similaridade de população e resultados, de forma que se caracterize a publicação científica associada à temática.

Foram realizadas buscas nas bases de dados LILACS e SciELO, com os seguintes descritores na língua portuguesa: osteoporose, exercício físico, idosos, tratamento e prevenção. Na base de dados PubMed procedeu-se a busca por meio dos seguintes descritores na língua inglesa: osteoporosis, physical exercise, elderly, prevention e treatment.

Foram critérios de exclusão:

1) artigos em outros idiomas que não os selecionados;

2) trabalhos científicos divulgados em outras formatações, configurados como revisões e materiais educativos;

3) artigos em meta-análise;

4) artigos com deficiência na descrição metodológica, principalmente no que se refere a objetivo, métodos e resultados. Ressalta-se ainda que se tomou a precaução necessária para que os artigos não fossem incluídos duas vezes, caso estivessem indexados nas bases de dados selecionadas.

\section{Resultados e discussões}

A partir das buscas realizadas nas bases de dados (LILACS, SciELO e PubMed), encontramos um total de 203 artigos. Esses estudos foram, na sua maioria, encontrados na base de dados PubMed (137 artigos), seguido da base LILACS (59 artigos) e SciELO (7 artigos). A partir dessa busca, ocorreu análise criteriosa, chegando a um total de 27 artigos selecionados. Esses foram divididos quanto ao método e objetivo do estudo referente ao tratamento e prevenção da osteoporose.

A maioria dos artigos selecionados (16 dos 27 trabalhos) tinha sua temática voltada para a prevenção da osteoporose. Observa-se também que a base de dados PubMed apresentou maior quantidade de estudos, tanto com relação à prevenção quanto ao tratamento, totalizando 21 dos artigos selecionados.

Atualmente, o exercício físico vem sendo utilizado no tratamento e na prevenção da osteoporose, e para que seja empregado da melhor forma, é necessário que o profissional tenha um conhecimento apurado sobre o efeito desse tipo de atividade na composição óssea dos idosos, pois eles podem apresentar ossos frágeis, o que pode levar (dependendo do tipo de exercício) ao risco de fratura (9). Observou-se, nesta pesquisa, que os exercícios mais estudados na literatura científica referente ao tratamento da osteoporose foram os de extensão isométrica de tronco (realizados em posição antigravitacional), exercícios em cadeia cinética aberta, corridas, caminhadas e exercícios de equilíbrio e coordenação (Tabela 1). Esses exercícios apresentaram benefícios múltiplos, como a diminuição da perda óssea, fortalecimento muscular, além da melhoria do equilíbrio, prevenindo assim futuras complicações causadas por quedas (10-12). 
Os exercícios de extensão isométrica de tronco, quando realizados em posição antigravitacional, são eficientes para a diminuição do risco de fraturas vertebrais, pois melhoram a densidade mineral óssea das vértebras e fortalecem a musculatura da região posterior do corpo, fato este que é muito importante, pois entre os idosos portadores de osteoporose há uma grande incidência de fraturas na região vertebral; sendo assim, esse tipo de exercício pode então prevenir possíveis fraturas no idoso osteoporótico. Os exercícios em cadeia cinética aberta atuam no fortalecimento dos músculos da coxa, são eficientes para a prevenção de fraturas, principalmente do colo femoral, e também ajudam a melhorar o condicionamento físico, elevando a qualidade de vida e fortalecendo a musculatura da região exercitada $(12,13)$. Exercícios como caminhadas mostraram-se pouco eficientes para a melhora da densidade mineral óssea, por esse tipo de exercício não exigir tanto dos ossos; ou seja, os ossos não são estressados a ponto de que sua massa aumente $(12,14$, 15). Sabe-se que exercícios como a corrida são eficientes para melhorar a composição da densidade mineral óssea, além de aprimorar o condicionamento físico e beneficiar outros sistemas, como, por exemplo, o cardiorrespiratório. Porém, quando é empregado como método de redução do risco de fraturas, esse exercício não apresenta resultados considerados eficientes (9). Exercícios de coordenação e equilíbrio são bastante utilizados no tratamento da osteoporose, principalmente por serem bastante eficientes no que se refere à melhoria tanto do equilíbrio dinâmico quanto do equilíbrio estático (Tabela 1). Esses exercícios diminuem os riscos de quedas e, posteriormente, de fraturas, que muitas vezes levam o idoso à morte $(10,15)$. Vale a pena ressaltar que os exercícios de coordenação e equilíbrio são eficazes apenas para a melhoria do equilíbrio, tanto dinâmico quanto estático, mas que não para o ganho de força muscular e melhoria da densidade mineral óssea $(10,15)$.

Tabela 1 - Estudos sobre exercício físico utilizados no tratamento da osteoporose segundo autor, tipo de exercício, objetivo, complementação e resultados dos exercícios

(continua)

\begin{tabular}{|c|c|c|c|c|}
\hline Autor/ano & Tipo de exercício & Objetivo do exercício & Complementação & Resultados \\
\hline $\begin{array}{l}\text { Bergstram; } \\
\text { Landgren; Brinck, } \\
\text { et al, } 2008^{(14)}\end{array}$ & Caminhadas rápidas & $\begin{array}{l}\text { Preservação da } \\
\text { densidade mineral } \\
\text { óssea em mulheres } \\
\text { osteoporóticas }\end{array}$ & Não houve & Pouco eficiente \\
\hline $\begin{array}{l}\text { Brooke-Wavell; } \\
\text { Prelevic; Bartram } \\
\text { et al, } 2000\end{array}$ & $\begin{array}{l}\text { Não especificou (medido } \\
\text { através de acelerômetro) }\end{array}$ & $\begin{array}{l}\text { Verificar se a atividade } \\
\text { física habitual } \\
\text { influencia na densidade } \\
\text { mineral de ossos } \\
\text { submetido à terapia de } \\
\text { substituição hormonal. }\end{array}$ & Não houve & $\begin{array}{l}\text { Quanto maior o } \\
\text { nível de atividade } \\
\text { física, maior a } \\
\text { densidade mineral } \\
\text { do osso. }\end{array}$ \\
\hline $\begin{array}{l}\text { Carter; Khan; Petit } \\
\text { et al, } 2001{ }^{(9)}\end{array}$ & $\begin{array}{l}\text { Corrida e exercício de } \\
\text { extensão de joelho. }\end{array}$ & $\begin{array}{l}\text { Reduzir os riscos de } \\
\text { queda }\end{array}$ & Não houve & Não eficiente \\
\hline $\begin{array}{l}\text { Granito; Renno; } \\
\text { Aveiro et al, } 2004 \text { (23) }\end{array}$ & $\begin{array}{l}\text { Não especificou exercício } \\
\text { - utilizou questionários: } \\
\text { Br-MPQ para avaliação } \\
\text { da dorsalgia e OPAQ } \\
\text { para avaliação da } \\
\text { qualidade de vida. }\end{array}$ & $\begin{array}{l}\text { Melhorar postura } \\
\text { hipercifótica torácica } \\
\text { (exame físico), } \\
\text { dorsalgia e qualidade } \\
\text { de vida }\end{array}$ & Não houve & Eficiente \\
\hline
\end{tabular}


Tabela 1 - Estudos sobre exercício físico utilizados no tratamento da osteoporose segundo autor, tipo de exercício, objetivo, complementação e resultados dos exercícios

(conclusão)

\begin{tabular}{|c|c|c|c|c|}
\hline Autor/ano & Tipo de exercício & Objetivo do exercício & Complementação & Resultados \\
\hline $\begin{array}{l}\text { Hongo; Itoi; Sinaki } \\
\text { et al, } 2007^{(11)}\end{array}$ & $\begin{array}{l}\text { Extensão isométrica } \\
\text { de tronco (posição } \\
\text { antigravitacional) }\end{array}$ & $\begin{array}{l}\text { Diminuir risco de } \\
\text { fraturas vertebrais, } \\
\text { fortalecer musculatura } \\
\text { extensora de troco e } \\
\text { melhorar qualidade } \\
\text { de vida }\end{array}$ & Não houve & Eficiente \\
\hline $\begin{array}{l}\text { Navega; Aveiro; } \\
\text { Oishi, } 2003{ }^{(13)}\end{array}$ & $\begin{array}{l}\text { Alongamentos gerais, } \\
\text { caminhada e exercícios } \\
\text { em cadeia cinética aberta }\end{array}$ & $\begin{array}{l}\text { Melhorar a tolerância } \\
\text { ao esforço físico, } \\
\text { equilíbrio, a } \\
\text { flexibilidade e a força } \\
\text { dos músculos extensores } \\
\text { e flexores do joelho }\end{array}$ & Não houve & Eficiente \\
\hline $\begin{array}{l}\text { Navega; Aveiro; } \\
\text { Oishi, } 2006^{(12)}\end{array}$ & $\begin{array}{l}\text { Cadeia cinética aberta } \\
\text { para fortalecimento dos } \\
\text { músculos extensores e } \\
\text { flexores do joelho }\end{array}$ & $\begin{array}{l}\text { Melhoria da qualidade } \\
\text { de vida }\end{array}$ & Não houve & Eficiente \\
\hline $\begin{array}{l}\text { Pearson; Burkhart; } \\
\text { Pifalo; Palaggo-Toy } \\
\text { et al, } 2005^{(21)}\end{array}$ & $\begin{array}{l}\text { Modificação do estilo de } \\
\text { vida quanto ao exercício } \\
\text { e nutrição }\end{array}$ & Prevenção de fraturas & Cálcio e vitamina D & Eficiente \\
\hline $\begin{array}{l}\text { Swanenburg; } \\
\text { Bruin; Stauffacher; } \\
\text { et al, } 2007^{(10)}\end{array}$ & $\begin{array}{l}\text { Treinamento de força } \\
\text { muscular, coordenação, } \\
\text { equilíbrio e resistência }\end{array}$ & $\begin{array}{l}\text { Diminuir os riscos de } \\
\text { queda, aumentar a força } \\
\text { muscular e o nível de } \\
\text { atividade física }\end{array}$ & Cálcio e vitamina D & $\begin{array}{l}\text { Muito eficiente } \\
\text { para diminuir } \\
\text { risco de quedas/ } \\
\text { eficientes para os } \\
\text { demais objetivos }\end{array}$ \\
\hline $\begin{array}{l}\text { Vaillant; Vuillerme } \\
\text { et al, } 2006^{(15)}\end{array}$ & $\begin{array}{l}\text { Exercícios de equilíbrio, } \\
\text { coordenação e } \\
\text { deambulação }\end{array}$ & $\begin{array}{l}\text { Reduzir os riscos de } \\
\text { queda }\end{array}$ & Não houve & Eficiente \\
\hline
\end{tabular}

A suplementação de cálcio e vitamina D tem grande importância no tratamento e na prevenção da osteoporose. Sabe-se que os ossos têm função de armazenamento de cálcio, e este, quando ingerido em quantidades adequadas, pode trazer grandes benefícios, principalmente para o idoso acometido pela osteoporose, pois o consumo aumenta sua concentração no sangue e nos ossos (16). Estima-se que 51\% do pico da massa óssea seja adquirido durante a puberdade, principalmente nas mulheres, sendo que $95 \%$ da quantidade total dos minerais ósseos depositam-se entre os 18 e os 22 anos, período em que o consumo de cálcio na dieta é muito importante para esse resultado (17). Estudos mostram que a maioria das mulheres com osteopenia consomem cálcio de forma inadequada e ainda apresentam pouca ou nenhuma prática de atividade física $(18,16)$. Por meio da contração muscular, o exercício físico transmite carga aos ossos, o que beneficia a densidade mineral destes. O consumo de vitamina $\mathrm{D}$ tem papel muito importante na absorção 
do cálcio pelo intestino e na reabsorção óssea, o que leva consequentemente a um aumento na quantidade de cálcio sérico. Faz-se necessária a exposição adequada à luz solar, pois os raios ultravioleta convertem a vitamina $\mathrm{D}$ em sua forma ativa no organismo $(19,20)$. Desse modo, podemos afirmar que o exercício físico, quando combinado com a suplementação de cálcio e vitamina $\mathrm{D}$, exerce efeitos bastante eficazes na melhoria da densidade mineral óssea $(10,16,21)$.

A prevenção da osteoporose pode se iniciar a partir da infância, principalmente com hábitos alimentares saudáveis e com a prática de atividade/exercício físico (19). Estudos mostram que esportes como o voleibol, quando praticados de modo competitivo a partir da segunda década de vida, e quando mantida a prática após a menopausa, exercem efeito bastante positivo na densidade mineral óssea durante a velhice (24). Entre os exercícios encontrados na literatura científica e que mais se destacaram quanto aos objetivos e resultados voltados à prevenção da osteoporose, podemos enfatizar os exercícios físicos de alta intensidade, exercícios aeróbios, exercícios de alto impacto e atividade física habitual. Esses exercícios e a atividade física habitual tiveram basicamente o objetivo de prevenir a perda da massa óssea, e a maioria apresentou resultados considerados muito eficientes, principalmente os exercícios de alta intensidade (Tabela 2). Foram encontrados outros exercícios, mas estes não demonstraram resultados tão eficientes.

Os exercícios de alta intensidade (futebol, corridas de velocidade, treinamento com pesos) mostraram-se eficientes na melhoria da composição óssea e também de outros fatores, como lipídios circulantes, aptidão física, além de diminuir os efeitos da menopausa (Tabela 2). Vale ressaltar que esses exercícios foram complementados com cálcio e vitamina $\mathrm{D}$ e foram realizados com mulheres osteopênicas $(25,26)$.

Tabela 2 - Apresentação entre tipos, objetivos e resultados dos exercícios utilizados na prevenção da osteoporose nos estudos selecionados

(continua)

\begin{tabular}{|c|c|c|c|c|}
\hline Autor/ano & Tipo de exercício & Objetivo do exercício & Complementação & Resultados \\
\hline $\begin{array}{l}\text { Brooke-Wavell; } \\
\text { Prelevic; Bartram } \\
\text { et al, } 2000\end{array}$ & $\begin{array}{l}\text { Não especificou (medido } \\
\text { através de acelerômetro) }\end{array}$ & $\begin{array}{l}\text { Verificar se a atividade } \\
\text { habitual física } \\
\text { influencia na densidade } \\
\text { mineral óssea quando } \\
\text { submetida à terapia de } \\
\text { substituição hormonal }\end{array}$ & Não houve & $\begin{array}{l}\text { Quanto maior o } \\
\text { nível de atividade } \\
\text { física, maior a } \\
\text { densidade mineral } \\
\text { óssea }\end{array}$ \\
\hline $\begin{array}{l}\text { Engelke; Kemmler; } \\
\text { Lauber et al, } 2006{ }^{(28)}\end{array}$ & $\begin{array}{l}\text { Exercício aeróbio de } \\
\text { força e de alta resistência } \\
\text { com baixo volume e de } \\
\text { alto impacto }\end{array}$ & $\begin{array}{l}\text { Prevenção da } \\
\text { osteoporose - } \\
\text { desacelerar perda óssea } \\
\text { na pós-menopausa }\end{array}$ & $\begin{array}{l}\text { Cálcio e vitamina } \\
\text { D }\end{array}$ & Eficiente \\
\hline Florindo, $2000^{(32)}$ & $\begin{array}{l}\text { Exercícios físicos } \\
\text { (medidos através de } \\
\text { questionário) nos } \\
\text { períodos de } 10 \text { a } 20 \text { anos } \\
\text { de idade, de } 21 \text { a } 30 \text { anos } \\
\text { de idade, de } 31 \text { a } 50 \text { anos } \\
\text { de idade e dos últimos } \\
12 \text { meses e atividades de } \\
\text { locomoção dos últimos } \\
12 \text { meses }\end{array}$ & $\begin{array}{l}\text { Verificar a relação do } \\
\text { nível de atividade física } \\
\text { ao longo da vida em } \\
\text { relação à densidade } \\
\text { mineral óssea }\end{array}$ & Estrógeno & $\begin{array}{l}\text { Exercícios físicos } \\
\text { praticados na } \\
\text { adolescência e as } \\
\text { atividades físicas } \\
\text { de locomoção } \\
\text { do cotidiano } \\
\text { melhoram a } \\
\text { composição } \\
\text { óssea e previne a } \\
\text { osteoporose }\end{array}$ \\
\hline
\end{tabular}


Tabela 2 - Apresentação entre tipos, objetivos e resultados dos exercícios utilizados na prevenção da osteoporose nos estudos selecionados

(continua)

\begin{tabular}{|c|c|c|c|c|}
\hline Autor/ano & Tipo de exercício & Objetivo do exercício & Complementação & Resultados \\
\hline $\begin{array}{l}\text { Huuskonen; Jurvelin; } \\
\text { Alhava et al, } 2001{ }^{(27)}\end{array}$ & $\begin{array}{l}\text { Treinamento de exercício } \\
\text { aeróbio regular }\end{array}$ & Diminuir a perda óssea & Não houve & Pouco eficiente \\
\hline $\begin{array}{l}\text { Ishikawa; Ohta; } \\
\text { Hirano et al, } 2000 \text { (17) }\end{array}$ & $\begin{array}{l}\text { Estilo de vida e número } \\
\text { de passos }\end{array}$ & $\begin{array}{l}\text { Prevenção da } \\
\text { osteoporose } \\
\text { pós-menopausa }\end{array}$ & $\begin{array}{l}\text { Verificou-se a } \\
\text { ingesta de cálcio, } \\
\text { leite e peixe }\end{array}$ & $\begin{array}{l}\text { Eficiente quando } \\
\text { seguido estilo de } \\
\text { vida saudável }\end{array}$ \\
\hline $\begin{array}{l}\text { Kemmler; von } \\
\text { Stengel; Weineck } \\
\text { et al } 2005^{(25)}\end{array}$ & $\begin{array}{l}\text { Exercício de alta } \\
\text { intensidade }\end{array}$ & $\begin{array}{l}\text { Melhorar composição } \\
\text { óssea do corpo, lipídios } \\
\text { circulantes, aptidão } \\
\text { física e sintomas da } \\
\text { menopausa - mulheres } \\
\text { com osteopenia }\end{array}$ & Cálcio e vitamina D & Muito eficiente \\
\hline $\begin{array}{l}\text { Kemmler, Lauber, } \\
\text { Weineck et al, } \\
2004^{(26)}\end{array}$ & $\begin{array}{l}\text { Exercício físico com } \\
\text { base na força máxima e } \\
\text { aptidão cardiovascular }\end{array}$ & $\begin{array}{l}\text { Melhorar a densidade } \\
\text { mineral óssea, aptidão } \\
\text { física e lipídios } \\
\text { circulantes e reduzir a } \\
\text { dor - mulheres com } \\
\text { osteopenia }\end{array}$ & Cálcio e vitamina D & Muito eficiente \\
\hline $\begin{array}{l}\text { Kemmler; } \\
\text { Engelke; Weineck } \\
\text { et al, } 2003^{(29)}\end{array}$ & $\begin{array}{l}\text { Exercício de alto impacto } \\
\text { combinado com força e } \\
\text { resistência }\end{array}$ & $\begin{array}{l}\text { Diminuir a perda } \\
\text { óssea - mulheres com } \\
\text { osteopenia }\end{array}$ & Cálcio e vitamina D & Eficiente \\
\hline $\begin{array}{l}\text { Kemmler; Engelke; } \\
\text { von Stengel et al, } \\
2007^{(30)}\end{array}$ & $\begin{array}{l}\text { Exercício aeróbicos } \\
\text { de alto impacto, saltos } \\
\text { laterais e multisséries de } \\
\text { resistência }\end{array}$ & $\begin{array}{l}\text { Diminuir os fatores de } \\
\text { riscos da osteoporose }\end{array}$ & Cálcio e vitamina D & Eficiente \\
\hline $\begin{array}{l}\text { Nguyen; Center; } \\
\text { Eisman, } 2000{ }^{(33)}\end{array}$ & Não especificou & $\begin{array}{l}\text { Verificar a relação } \\
\text { entre o índice de } \\
\text { atividade física, ingesta } \\
\text { de cálcio e densidade } \\
\text { mineral óssea }\end{array}$ & Cálcio & $\begin{array}{l}\text { Eficiente quando } \\
\text { se tem uma boa } \\
\text { ingesta diária de } \\
\text { cálcio e bom índice } \\
\text { de atividade física }\end{array}$ \\
\hline $\begin{array}{l}\text { Park; Togo } \\
\text { Watanabe et al, } \\
2007^{(31)}\end{array}$ & $\begin{array}{l}\text { Atividade física habitual - } \\
\text { Número de passos } \\
\text { (medido através de } \\
\text { acelerômetro) }\end{array}$ & Melhorar saúde óssea & Não houve & Não eficiente \\
\hline $\begin{array}{l}\text { Shirazi; Wallace; } \\
\text { Niknami et al, } \\
2007 \text { (34) }\end{array}$ & $\begin{array}{l}\text { Programa educacional } \\
\text { de Exercícios de força e } \\
\text { equilíbrio e caminhada }\end{array}$ & $\begin{array}{l}\text { Prevenção da } \\
\text { osteoporose }\end{array}$ & Não houve & Eficiente \\
\hline
\end{tabular}


Tabela 2 - Apresentação entre tipos, objetivos e resultados dos exercícios utilizados na prevenção da osteoporose nos estudos selecionados

(conclusão)

\begin{tabular}{lllll}
\hline \multicolumn{1}{c}{ Autor/ano } & \multicolumn{1}{c}{ Tipo de exercício } & Objetivo do exercício & Complementação & Resultados \\
\hline $\begin{array}{l}\text { Snelling; Crespo; } \\
\text { Schaeffer et al, } \\
2001^{(35)}\end{array}$ & $\begin{array}{l}\text { Fatores modificáveis: } \\
\text { prática regular de exercícios } \\
\text { (três a cinco vezes semanais) } \\
\text { composição corporal }\end{array}$ & $\begin{array}{l}\text { Prevenção da } \\
\text { osteoporose }\end{array}$ & Não houve & Eficiente \\
$\begin{array}{l}\text { Snow; Shaw; Winters } \\
\text { et al, } 2000^{(36)}\end{array}$ & $\begin{array}{l}\text { Exercício em longo prazo, } \\
\text { utilizando vestimenta } \\
\text { com peso }\end{array}$ & $\begin{array}{l}\text { Prevenção de fraturas } \\
\text { de quadril }\end{array}$ & Não houve & Eficiente \\
\hline
\end{tabular}

Os exercícios aeróbios também possuem efeitos eficientes na prevenção da osteoporose, quando combinados com exercícios de força, de alta resistência e complementados com a ingestão de cálcio e vitamina D. Quando são praticados sem combinação e de forma regular, não apresentam eficiência na prevenção da perda da massa óssea. Dessa forma, é importante ressaltar que, apesar dos exercícios aeróbios serem considerados pouco eficientes pela literatura científica, por não demonstrarem um aumento significativo da massa magra, não é possível avaliar esse tipo de exercício como uma modalidade terapêutica contraindicada, e sim uma modalidade que não deve ser empregada de maneira isolada na prevenção e tratamento da osteoporose $(27,28)$.

Quanto à prevenção da perda óssea, os exercícios de alto impacto mostraram-se também eficientes, pois esse tipo de exercício exige maior resistência óssea, expondo, assim, esses ossos a episódios de estresse o que por um lado causa efeitos positivos quanto à rigidez desses ossos, mas que por outro lado pode causar fraturas quando eles são expostos a extremo grau de estresse $(29,30)$. A atividade física habitual foi avaliada, na maioria dos estudos selecionados, por meio do número de passos dados, medidos por um acelerômetro. Esse tipo de atividade não apresentou resultados considerados eficientes para a melhora da composição óssea, porém apresentou efeitos positivos apenas quando se adquire hábitos saudáveis como o consumo de cálcio, comprovando, assim, a influência benéfica de uma alimentação saudável tanto na prevenção quanto no tratamento da osteoporose $(17,31)$.

Desse modo, podemos afirmar que a prevenção da osteoporose por meio de exercícios físicos pode ser realizada desde as fases da infância até a velhice, pois a manutenção de hábitos de vida saudáveis como a prática de exercícios físicos tem grande parcela de contribuição para a diminuição de possíveis complicações provenientes da velhice (32).

\section{Considerações finais}

A partir dos dados obtidos nesta revisão podemos concluir que o exercício físico é dado como fator importante tanto na prevenção quanto no tratamento da osteoporose. A maioria dos estudos apresentou temática voltada à prevenção, o que mostra a ênfase dada pela comunidade científica para evitar a osteoporose. Foi possível verificar que os exercícios possuem especificidades, dependendo do objetivo com que são empregados, pois, como visto no corpo do trabalho, os exercícios utilizados no tratamento da osteoporose foram caracterizados como de baixa e média intensidade - uma vez que os ossos do idoso acometido pela osteoporose podem ser fraturados se empregados exercícios intensos e que produzam grandes impactos. Pôde-se também observar que os exercícios que agem na melhora do equilíbrio e coordenação são eficientes 
no tratamento, pois podem reduzir os riscos de quedas que poderiam vir a causar fraturas para o acometido pela doença. Também se observou que os exercícios utilizados na prevenção da osteoporose foram caracterizados como de alta intensidade, por aumentarem significativamente a densidade mineral óssea e porque como na prevenção a pessoa não apresenta osteoporose, é menor o risco de fraturas. Como fator contribuinte, a manutenção de hábitos alimentares saudáveis exerce efeitos benéficos, tanto na prevenção quanto no tratamento de idosos com osteoporose.

\section{Agradecimentos}

À Fundação de Amparo à Pesquisa do Estado do Amazonas (FAPEAM), pela Bolsa de Iniciação Científica.

\section{Referências}

1. Frazão P, Naveira M. Prevalência de osteoporose: uma revisão crítica. Revista Brasileira de Epidemiologia. 2006;9:206-214.

2. Carvalho JAM, Garcia RAG. O envelhecimento da população brasileira um enfoque demográfico. Cad Saúde Pública. 2003;19(3):725-33.

3. Navega MT. Comparação da qualidade de vida relacionada à saúde entre mulheres na pós-menopausa praticantes de atividade física com e sem osteoporose. Rev Bras Reumatol. 2007;47(4):258-64.

4. Hebert S, Xavier R. Ortopedia e traumatologia: princípios e prática. 3a ed. Porto Alegre: Artmed; 2003.

5. Lemos MCD, Miyamoto, S.T, Valim, V, Natour J. Qualidade de vida em pacientes com osteoporose: correlação entre OPAQ e SF-36. Rev Bras Reumatol. 2006;46(5):323-8.

6. Gali JC. Osteoporose. Acta ortopédica Brasileira. 2001;9(2):53-62.

7. Ocarino NM. Efeito da atividade física no osso normal e na prevenção e tratamento da osteoporose. Rev Bras Med Esporte. 2006;12(3):164-8.

8. Colégio Americano de Medicina do Esporte. Posicionamento Oficial do Colégio Americano de Medicina do Esporte sobre Osteoporose e Exercício. Med Sci Sports Exerc. 1995;27(4):1-7.

9. Carter ND, Khan KM, Petit MA, Heinonen A, Waterman C, Donaldson MG, et al. Results of a 10 week community based strength and balance training programme to reduce fall risk factors: a randomised controlled trial in 65-75 year old women with osteoporosis. Br J Sports Med. 2001;35(5):348-51.

10. Swanenburg J, de Bruin ED, Stauffacher M, Mulder T, Uebelhart D. Effects of exercise and nutrition on postural balance and risk of falling in elderly people with decreased bone mineral density: randomized controlled trial pilot study. Clin Rehabil. 2007;21(6):523-34.

11. Hongo M, Itoi E, Sinaki M, Miyakoshi N, Shimada Y, Maekawa S, et al. Effect of low-intensity back exercise on quality of life and back extensor strength in patients with osteoporosis: a randomized controlled trial. Osteoporos Int. 2007;18(10):1389-95.

12. Navega MT, Aveiro MC, Oishi J. A influência de um programa de atividade física na qualidade de vida de mulheres com osteoporose / The influence of a physical exercise program on the quality of life in osteoporotic women. Fisioter Mov. 2006;19(4):25-32.

13. Navega MT, Aveiro MC, Oishi J. Alongamento, caminhada e fortalecimento dos músculos da coxa: um programa de atividade fisica para mulheres com osteoporose / Stretching, walking and strenghtening of thigh muscles: a physical activity program to osteoporotic women. Rev Bras Fisioter. 2003;7(3):261-7. 
14. Bergstrãm I, Landgren B, Brinck J, Freyschuss B. Physical training preserves bone mineral density in postmenopausal women with forearm fractures and low bone mineral density. Osteoporos Int. 2008;19(2):177-83.

15. Vaillant J, Vuillerme N, Martigné P, Caillat-Miousse JL, Parisot J, Nougier V, et al. Balance, aging, and osteoporosis: effects of cognitive exercises combined with physiotherapy. Joint Bone Spine. 2006;73(4):414-8.

16. Lanzillotti HS, Lanzillotti RS, Trotte APR, Dias AS, Bornand B, Costa EAMM. Osteoporose em mulheres na pós-menopausa, cálcio dietético e outros fatores de risco. Rev Nutrição. 2003;16(2):181-193.

17. Ishikawa K, Ohta T, Hirano M, Yoshimoto K, Tanaka S, Inoue S. Relation of lifestyle factors to metacarpal bone mineral density was different depending on menstrual condition and years since menopause in Japanese women. Eur J Clin Nutr. 2000;54(1):9-13.

18. Brandão CMR, Lima MG, Silva AL, Silva GD, Guerra AA, Acúrcio FA. Tratamento da osteoporose em mulheres na pós-menopausa: uma revisão sistemática. Cad. Saúde Pública. 2008;24(supl 4):s592-s606.

19. Morais GQ, Burgos MGP. Impacto dos nutrientes na saúde óssea: novas tendências. Nutrients impact on bone health: new trends. Rev Bras Ortop. 2007;42(7):189-94.

20. Russo LAT. Osteoporose Pós-Menopausa: Opções Terapêuticas. Arq Bras Endocrinol Metab. 2001;45(4):401-6.

21. Pearson JA, Burkhart E, Pifalo WB, Palaggo-Toy T, Krohn K. A lifestyle modification intervention for the treatment of osteoporosis. Am J Health Promot. 2005;20(1):28-33.

22. Brooke-Wavell K, Prelevic GM, Bartram C, Ginsburg J. The influence of physical activity on the response of bone mineral density to 5 years tibolone. Maturitas. 2000;35(3):229-35.

23. Granito RN, Renno ACM, Aveiro MC, Navega MT, Driusso P, Oishi J. Efeitos de um programa de atividade física na postura hipercifótica torácica, na dorsalgia e na qualidade de vida de mulheres com osteoporose. Rev Bras Fisioter. 2004;8(3):231-7.

24. Karam FC, Meyer F, Souza ACA. Esporte como prevenção de osteoporose: um estudo da massa óssea de mulheres pós-menopáusicas que foram atletas de voleibol / Sports preventing osteoporosis: bone mass in postmenopausal women who played volleyball. Rev Bras Med Esporte. 1999;5(3):86-92.

25. Kemmler W, von Stengel S, Weineck J, Lauber D, Kalender W, Engelke K. Exercise effects on menopausal risk factors of early postmenopausal women: 3-yr Erlangen fitness osteoporosis prevention study results. Med Sci Sports Exerc. 2005;37(2):194-203.

26. Kemmler W, Lauber D, Weineck J, Hensen J, Kalender W, Engelke K. Benefits of 2 years of intense exercise on bone density, physical fitness, and blood lipids in early postmenopausal osteopenic women: results of the Erlangen Fitness Osteoporosis Prevention Study (EFOPS). Arch Intern Med. 2004;164(10):1084-91.

27. Huuskonen J, Jurvelin JS, Alhava E, Rauramaa R. Regular physical exercise and bone mineral density: a four-year controlled randomized trial in middle-aged men. The DNASCO study. Osteoporos Int. 2001;12(5):349-55.

28. Engelke K, Kemmler W, Lauber D, Beeskow C, Pintag R, Kalender WA. Exercise maintains bone density at spine and hip EFOPS: a 3-year longitudinal study in early postmenopausal women. Osteoporos Int. 2006; 17(1):133-42.

29. Kemmler W, Engelke K, Weineck J, Hensen J, Kalender WA. The Erlangen Fitness Osteoporosis Prevention Study: a controlled exercise trial in early postmenopausal women with low bone density-first-year results. Arch Phys Med Rehabil. 2003;84(5):673-82.

30. Kemmler W, Engelke K, von Stengel S, Weineck J, Lauber D, Kalender WA. Long-term four-year exercise has a positive effect on menopausal risk factors: the Erlangen Fitness Osteoporosis Prevention Study. J Strength Cond Res. 2007;21(1):232-9. 
31. Park H, Togo F, Watanabe E, Yasunaga A, Park S, Shephard RJ, et al. Relationship of bone health to yearlong physical activity in older Japanese adults: cross-sectional data from the Nakanojo Study. Osteoporos Int. 2007;18(3):285-93.

32. Florindo AA. Atividade física habitual e densidade mineral óssea em homens adultos e idosos / Habitual physical activity and bone mineral density in men aged 50 years and older. Rev Bras Ativ Fís Saúde. 2000;5:22-34.

33. Nguyen TV, Center JR, Eisman JA. Osteoporosis in elderly men and women: effects of dietary calcium, physical activity, and body mass index. J Bone Miner Res. 2000;15(2):322-31.

34. Shirazi KK, Wallace LM, Niknami S, Hidarnia A, Torkaman G, Gilchrist M, et al. A home-based, transtheoretical change model designed strength training intervention to increase exercise to prevent osteoporosis in Iranian women aged 40-65 years: a randomized controlled trial. Health Educ Res. 2007;22(3):305-17.

35. Snelling AM, Crespo CJ, Schaeffer M, Smith S, Walbourn L. Modifiable and non-modifiable factors associated with osteoporosis in postmenopausal women: results from the Third National Health and Nutrition Examination Survey, 1988-1994. J Womens Health Gend Based Med. 2001;10(1):57-65.

36. Snow CM, Shaw JM, Winters KM, Witzke KA. Long-term exercise using weighted vests prevents hip bone loss in postmenopausal women. J Gerontol A Biol Sci Med Sci. 2000;55(9):M489-91.

Recebido: 08/04/2009

Received: 04/08/2009

Aprovado: $23 / 03 / 2010$

Approved: 03/23/2010

Revisado: $13 / 05 / 2010$

Reviewed: 05/13/2010 